\title{
La necesaria actualización de los Códigos UNESCO en Antropología Social en el siglo XXI
}

\section{The necessary updating of the UNESCO Codes in Social Anthropology in the $21^{\text {st }}$ century}

\author{
Klaus SCHRIEWER \\ Juan Ignacio RICO BECERRA \\ Universidad de Murcia *
}

\begin{abstract}
Resumen: En este texto pretendemos poner de manifiesto por qué es imprescindible actualizar los Códigos UNESCO en Antropología Social. Para tal fin realizamos, en primer lugar, una revisión retrospectiva de los Códigos UNESCO partiendo del primer borrador de 1972 hasta la actualidad y de sus limitaciones. Después exponemos las valoraciones de los antropólogos/as españoles/as que han participado en nuestra encuesta sobre la adecuación de los Códigos UNESCO; y finalmente, elaboramos una propuesta de los Códigos UNESCO para Antropología.
\end{abstract}

Palabras clave: Códigos UNESCO; encuesta de antropólogos/as; clasificación de campos científicos; campo antropológico; disciplina; subdisciplina.

Abstract: In this text we intend to highlight why it is essential to update the UNESCO Codes for Social Anthropology. To this end we carried out, in the first place, a retrospective review of the UNESCO Codes and its limitations, starting from the first draft of 1972 to the present. Then we expose the valuations of the Spanish anthropologists who have participated in our survey on the adequacy of the UNESCO Codes, and finally we present a proposal of the UNESCO Codes for Anthropology.

Key words: UNESCO nomenclature; survey among anthropologists; classification of scientific fields; anthropological field; discipline; sub-discipline.

\footnotetext{
* Recibido: 8, septiembre, 2018. Aceptado: 10, octubre, 2018.

Email: ks@um.es; juan.rico@um.es.

http://dx.doi.org/10.6018/rmu/355551
} 


\section{INTRODUCCIÓN}

El documento Presente y futuro de la Antropología Social y Cultural española: A partir de las reflexiones sobre niveles de asociación científico-académica entre áreas de conocimiento, que encabeza los artículos que se presentan a continuación, tiene como objetivo fomentar el debate sobre el papel de nuestra disciplina tanto en el mundo académico como en la sociedad y, con ello, lo relativo a la identidad de la Antropología. No es esta una reflexión que haya estado ausente en nuestra disciplina, como demuestra, por ejemplo, el acto llevado a cabo sobre la historia y las perspectivas de la Antropología en el último congreso de la FAAEE en Valencia, ${ }^{1}$ pero con todo, nos parece de interés seguir haciendo hincapié en algunos elementos que consideramos indispensables para poder afrontar los nuevos retos que se presentan en la actualidad.

Nos propusimos revisar diferentes aspectos que están en la órbita del cometido propuesto: los llamados Códigos UNESCO como marco internacionalmente aplicado para la definición de las disciplinas académicas, y el decurso de la codificación de Antropología; la relación entre esta y las disciplinas afines en el conjunto académico; y, finalmente, la valoración de los antropólogos sobre las perspectivas de nuestra disciplina. En este primer artículo tratamos la definición de la Antropología que ofrece el «International Standard Nomenclature for Fields of Science and Technology», conocido como Códigos UNESCO, que tiene un impacto de hondo calado en la vida académica española. Nuestro propósito es debatir sobre su adecuación y actualidad, así como presentar una primera propuesta para su actualización.

Para ello, como indicábamos, vamos a comenzar con una breve revisión de los orígenes de esta nomenclatura, la definición de los términos utilizados y la aplicación de los Códigos UNESCO en España; en la segunda parte presentamos las valoraciones de antropólogos/as españoles/as que han participado en nuestra encuesta sobre la adecuación de los Códigos UNESCO; y en la tercera, elaboramos, basándonos en estas aportaciones y anteriores reflexiones, una primera propuesta para el debate sobre una modificación de los Códigos UNESCO en Antropología.

1 «La historia de la Antropología y la Etnología en España y el mundo Hispanoamericano», organizado por Andrés Barrera González y Carmen Ortiz García el 6 de septiembre 2017 en el Congreso de la FAAEE: «Antropologías en transformación. Sentidos, compromisos y utopías». 


\section{LOS CÓDIGOS UNESCO}

Los Códigos UNESCO son sin duda un instrumento que ha influido con gran impacto en la estructuración del mundo académico internacional y, por ende, en el español. Se utilizan para muchos aspectos de la vida académica. Es el caso de las convocatorias de educación para profesorado, la clasificación del campo donde se adscriben las tesis doctorales o la sistematización de las actividades de grupos de investigación, entre otros. Se trata, en definitiva, de una de las principales herramientas utilizadas en el diseño del mundo académico.

Considerando, como decíamos, que estos Códigos han pergeñado la sistematización del ámbito académico que hoy conocemos, nos preguntamos si la articulación actual de los Códigos UNESCO sigue siendo adecuada. Es conocido que muchas de las disciplinas han evolucionado en las últimas décadas, gracias al propio desarrollo de las disciplinas, la introducción de las nuevas tecnologías y los cambios sociopolíticos. La cuestión entonces es si los Códigos UNESCO se han actualizado considerando tales variaciones. Y no parece que esto haya sido así, como muestra, por ejemplo, la valoración de Ruiz Martínez, Baños Moreno y Martínez Béjar: «La ruptura con la realidad científica y tecnológica es evidente, puesto que las nuevas disciplinas y ramas surgidas en los últimos años no están contempladas en la clasificación» ${ }^{2}$ Partiendo de la premisa anterior, para seguir indagando, decidimos revisar primero la evolución de los Códigos UNESCO.

La iniciativa de crear una nomenclatura de las disciplinas académicas nace en la década de los 60 del siglo pasado como respuesta a una globalización que se acelera en esos años. En un primer documento, la Oficina de Estadística de la UNESCO anuncia que se va a conseguir «after some years perhaps the formulation of a recommendation for an international standard in this field». ${ }^{3}$ El objetivo vinculado con los Códigos es crear una herramienta que permita elaborar estadísticas comparativas que pueden servir para la gestión y las políticas en el ámbito de las ciencias y la tecnología. En 1972 la UNESCO publica un primer documento borrador que posteriormente experimenta cambios profundos. ${ }^{4}$ Un año más

2 J. M. Ruiz Martínez; M. J. Baños Moreno \& R. Martínez BéJar: «Nomenclatura Unesco. Evolución, alcance y reutilización en clave ontológica para la descripción de perfiles científicos», $E l$ profesional de la información, 23, n. 4, 2014, pp. 383-392, p. 384.

3 UNESCO: Problems Encountered in the Development of a Standard International Methodology of Science Statistics, Meeting of the Advisory Panel on Science Statistics, UNESCO/CS/0666.SS-80/5, Paris, 1966, p. 4.

4 UNESCO: Provisional List of Scientific Disciplines, UNESCO/NS/ROU/257, Paris: March 1972. 
tarde, en 1973, se da a conocer la propuesta de la nomenclatura ${ }^{5}$ que posteriormente se aplica en España. Pero no es hasta 1988 cuando se publica el documento definitivo denominado Proyecto de Nomenclatura Internacional Normalizada relativa a la Ciencia y la Tecnología 6 que, con algunas modificaciones menores, se aplica hasta la actualidad. Quepa resaltar para el debate que nos ocupa, que la propia UNESCO, tal como apuntan Ruiz Martínez, Baños Moreno y Martínez Béjar abandona el proyecto en $1992 .{ }^{7}$

En nuestro país hay que esperar hasta 1983 para que, por Resolución de 23 de septiembre de 1983 (BOE de 14 de octubre), la propuesta de 1973 pase a ser la clasificación utilizada por el Ministerio de Ciencia y Tecnología del Estado español. ${ }^{8}$ A partir de aquí el Consejo Superior de Investigaciones Científicas ha hecho públicas sucesivas modificaciones por Acuerdos de su Junta de Gobierno a través de las resoluciones de 28 de marzo de 1985, 25 de marzo de 1986 y 10 de marzo de 1988. Podemos decir que los Códigos UNESCO siguen influyendo en nuestra legislación. Así, el Real Decreto 1312/2007 establece un sistema que recuerda a las clasificación de la UNESCO, aunque de una forma modificada.

En cuanto a los términos utilizados, la estructura terminológica es la siguiente: campos, disciplinas y subdisciplinas. A saber:

1. Campos. Se refieren a los apartados más generales. Están codificados en dos dígitos y comprende varias disciplinas.

2. Disciplinas. Suponen una descripción general de grupos de especialidades en Ciencia y Tecnología. Son apartados codificados con cuatro dígitos. A pesar de ser distintas entre sí las disciplinas con referencias cruzadas, o dentro de un mismo campo, se considera que tienen características comunes.

3. Subdisciplinas: Son las entradas más específicas de la nomenclatura y representan las actividades que se realizan dentro de una disciplina. Están codificadas con seis dígitos. A su vez, deben corresponderse con las especialidades individuales en Ciencia y Tecnología.

5 UNESCO: Proposed International Standard Nomenclature for Fields of Science and Technology, UNESCO NS/ROU/257 rev. 1, Paris: 17 December 1973.

6 UNESCO: Proyecto de Nomenclatura Internacional Normalizada relativa a la Ciencia y la Tecnología, UNESCO NS/ROU/257 rev. 1, París: 17 de diciembre 1988.

7 J. M. Ruiz Martínez; M. J. Baños Moreno \& R. Martínez BéJar: «Nomenclatura Unesco...», 2014 , p. 385.

8 Ministerio de Ciencia, Innovación y Universidades del Gobierno de España: < http://www.idi. mineco.gob.es/portal/site/MICINN/menuitem.8ce192e94ba842bea3bc811001432ea0/?vgnextoid=363ac9487fb02210VgnVCM1000001d04140aRCRD\&vgnextchannel=28fb282978ea0210Vgn VCM1000001034e20aRCRD>. 
Estos tres niveles han experimentado varias modificaciones que en relación con la disciplina Antropología son de gran interés.

\subsection{Los campos}

Respecto a los campos hay que señalar que «Antropología» no ha sido reconocida como un campo académico propio al principio de la elaboración de la nomenclatura. El primer borrador de 1972 no valida Antropología como un campo propio. Tampoco lo hace en una primera lista, al principio del documento, ni en la relación más detallada que contiene. Subordina Antropología como disciplina de cuatro dígitos, al campo Sociología (32). Ya la propuesta de 1973 contiene un cambio muy profundo, elevando Antropología a un campo propio (fuera de la órbita de Sociología) y otorgándole los dígitos 51.

Más allá de la clasificación con dos dígitos que señalan los campos, el primer borrador diferencia cinco ámbito parecidos a las ramas que conocemos en el sistema actual español, que denomina: Ciencias exactas, Física y Ciencias naturales, Ciencias aplicadas, Ciencias humanas y sociales, así como, un ámbito denominado Generalidades. ${ }^{9}$ Es destacable que este primer borrador todavía presenta las Humanidades y Ciencias sociales como un conjunto, si bien excluye Filosofía como una de las llamadas Generalidades. Hay que tener en cuenta que las posteriores versiones de los Códigos UNESCO dejan de mencionar de manera explícita las ramas, aunque sí ordenan los campos según los decimales. Si en la actualidad en distintas universidades españolas se barajan documentos que clasifican los campos establecidos por la UNESCO según las ramas que manejamos, son interpretaciones propias que no se pueden deducir de los Códigos UNESCO.

El documento de 1988 categoriza Lógica y Matemáticas bajo los decimales 1, y disciplinas relacionadas con las Ciencias naturales en el grupo de decimales 2. En el grupo que comienza con el dígito 3 se encuentran las disciplinas aplicadas, incluyendo las de la salud. En este documento el decimal 4 desaparece, algo que señala hacia las profundas modificaciones que experimenta el sistema. Es a partir del decimal 5 donde aparecen las disciplinas que potencialmente pueden tener una relación más estrecha con la Antropología. Bajo el decimal 5 se recogen campos que, bajo nuestro criterio, pertenecerían tanto a las Ciencias sociales como a las Humanidades: Antropología (51) aparece junto con Demografía (52), Economía (53), Geografía (54), Historia (55), Ciencias jurídicas y Derecho (56), Lingüística (57), Pedagogía (58), y Ciencias políticas (59). En el siguiente campo, que comienza con el dígito 6, están subsumidos Psicología (61), Artes y Letras

9 UNESCO: Provisional List of Scientific Disciplines... 1972, p. 2. 
(62) y Sociología (63). El último campo comprende Ética (71) y Filosofía (72). Es evidente que los que contienen los decimales 5, 6 y 7 no siguen una lógica que corresponda actualmente en España con las ramas aplicadas. Lo que quizás se produzca por la falta de diferenciación entre las Humanidades y las Ciencias sociales. De hecho, en España posteriormente se abre la posibilidad de incorporarlos en ambos campos. Es decir, se ha abierto la posibilidad de integrar algunas de las disciplinas a la vez en ambas ramas (Humanidades y Ciencias sociales). De igual forma ha pasado con Antropología social y Geografía humana.

\subsection{Las disciplinas}

Las disciplinas se establecen desde clasificaciones que se constituyen a partir de cuatro dígitos. Establecen una red de disciplinas dentro de los campos. En el caso de Antropología, en el primer borrador de 1972, podemos observarla como parte de una disciplina que se inserta en la órbita de Sociología (32) y que encasilla la labor antropológica bajo la denominación Cultural/social, anthropology, ethnology ${ }^{10}$ con el código 3208. La propuesta de 1973, como ya hemos comentado, eleva Antropología a un campo propio, lo que lleva consigo la definición de disciplinas dentro de este nuevo campo. Estas disciplinas señaladas con cuatro dígitos, se generan dividiendo la disciplina antropológica del anterior borrador en tres, a saber: Antropología cultural (5101), Etnología y Etnografía (5102), y Antropología social (5103). Además de estas tres disciplinas se introduce la categoría «Otras Especialidades Antropológicas (especificar)» bajo el código 5199. ${ }^{11}$ Reconociendo así sus limitaciones, señala que el sistema está diseñado para experimentar modificaciones. Asimismo se hace referencia a la disciplina Antropología física (2402), ubicada en el campo Ciencias de la vida (24).

Se puede deducir del documento de la UNESCO de 1972 que la diferenciación fijada fue elaborada a partir de las categorías del mundo académico estadounidense, donde se distinguía entre la tradición británica y americana. Luego no refleja el debate que en estos momentos se estaba llevando a cabo en la Europa continental; ni la división entre las dos antropologías, que caracteriza la labor antropológica en Europa. ${ }^{12}$ Cabe preguntarse por la conveniencia de mantener la diferenciación entre las tres disciplinas antropológicas, y esto especialmente si tenemos que especificar diferentes subdisciplinas en cada una de ellas. El dilema

10 UNESCO: Provisional List of Scientific Disciplines... 1972, p. 17.

11 UNESCO: Proposed International Standard Nomenclature... 1973, p. 16.

12 Th. SCHIPPERS: «A history of paradoxes. Anthropologies of Europe», en H. Vermeulen \& A. Álvarez Roldán (eds.): Fieldworks and Footnotes. Studies $i$ the History of European Anthropology, London: Routledge, 1995, pp. 234-246. 
que aparece se puede mostrar con un ejemplo: ¿La subdisciplina Antropología urbana o Antropología de la migración formaría parte de Antropología cultural, de Etnología y Etnografía o de Antropología social? Y esto nos llevaría a otro cuestionamiento de mayor calado, ¿es posible sistematizar la labor antropológica mediante estas tres supuestas disciplinas? A lo visto, la revisión de las subdisciplinas incrementa las dudas.

\subsection{Las subdisciplinas}

La lista de subdisciplinas recogidas con seis dígitos ofrece la descripción más detallada de la actividad antropológica que encontramos en los Códigos UNESCO. Nos permite revisar con detalle si, y en qué medida, refleja la actividad antropológica. También el diseño de las subdisciplinas ha experimentado varios cambios que nos permiten averiguar cómo se ha intentado crear una sistemática adecuada.

El primer borrador de 1972 nos ofrece una larga lista con diecinueve subdisciplinas, ${ }^{13}$ que de una manera muy genérica intenta recoger la labor antropológica y refleja las temáticas abordadas en los años 1970. Es palmario que la descripción está inspirada en la tradición antropológica vinculada con el estudio de las llamadas sociedades simples, no-europeas. También es destacable que las versiones posteriores no incluyen todas las subdisciplinas que alista este borrador, o las trasladan a otro campo. Es el caso, como exponemos después, de la Antropología médica, que aparece en esta primera relación, ver abajo el código 3208.11, y que en la siguiente clasificación se ubicará en el campo Ciencias de la vida, en la disciplina Antropología física con el código 2402.07.

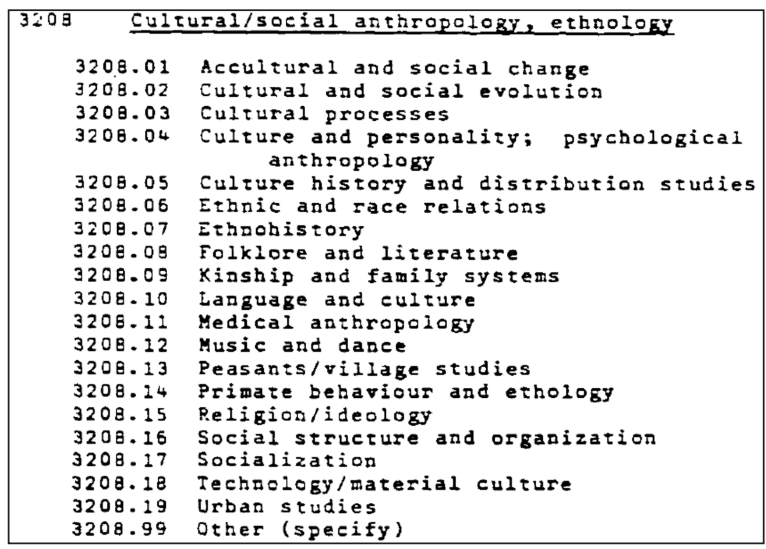

Ilustración 1: Subdisciplinas antropológicas del borrador de 1972.

13 UNESCO: Provisional List of Scientific Disciplines... 1972. 
La propuesta de los Códigos UNESCO que se presenta un año más tarde (1973), ${ }^{14}$ contiene una profunda modificación y especificación respecto a la primera (1972). Y se introduce sin modificaciones en el documento de 1988.15 Enumera una serie de treinta subdisciplinas antropológicas y las reparte en las tres disciplinas antropológicas: Antropología cultural, Etnología y Etnografía, y Antropología social. El resultado es una sistemática que parece algo artificial y que no concuerda con la actual realidad en España. La que lleva la denominación Antropología social utilizada en España y que aglutina toda la labor antropológica, está orientada al estudio de los siguientes temas: Jefatura y realeza (5103.01), Descendencia, familia y linaje (5103.02), Nomadismo (5103.03), Esclavitud y servidumbre (5103.04) y Guerra (5103.05). Además hay, como siempre, un campo denominado «Otros (especificar)» que lleva el código 5103.99 .

\section{Antropologia social \\ 5103.01 Jefatura, realeza \\ 5103.02 Descendencia, familia, Tinaje \\ 5103.03 Nomadismo \\ 5103.04 Esclavitud, servidumbre \\ 5103.05 Guerra (véase 6304.03) \\ 5103.99 Otros (especificar)}

Ilustración 2: Subdisciplinas de Antropología social en las propuestas de 1973 y 1988.

Es evidente que estas subdisciplinas se refieren casi en su totalidad al estudio de las llamadas sociedades simples y no tienen mucha relación con las tareas a las que se están dedicando los antropólogos en la actualidad. Muy similar es el enfoque de las once subdisciplinas que conforman la disciplina Etnología y Etnografía. Así, subdisciplinas como Caza, Pesca, y especialmente Trueque e Intercambio, demuestran que se mantiene en una visión tradicional de la Antropología.

14 UNESCO: Proposed International Standard Nomenclature... 1973.

15 UNESCO: Proyecto de Nomenclatura Internacional Normalizada... 1988. 


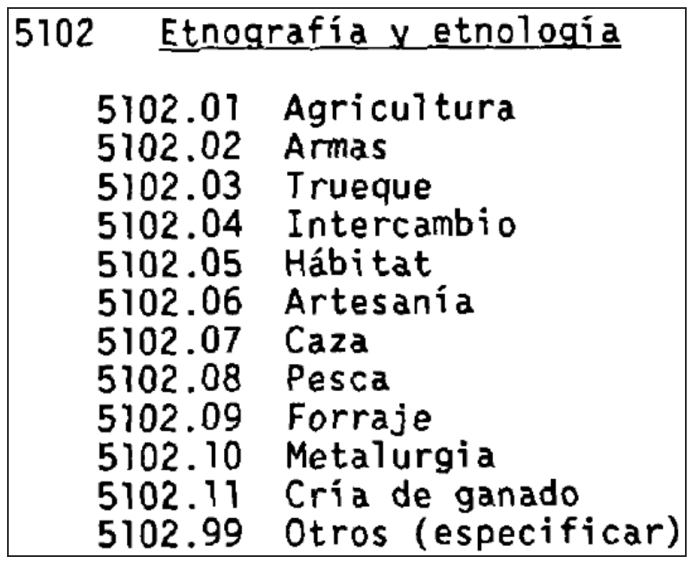

Ilustración 3: Subdisciplinas de Etnología y Etnografía en las propuestas de 1973 y 1988.

La tercera disciplina que menciona el documento, Antropología cultural, está compuesta por catorce subdisciplinas que en algunos casos son muy generales, como Religión o Simbolismo, y por ello, tienen mayor correspondencia con campos actuales. No obstante, también esta relación se caracteriza por una visión tradicional al dirigir su orientación a temas como Adorno, Vestimenta, Danzas, etc., que aún formando parte de los objetos de estudio antropológico, no ocupan un lugar destacado como aquí se surgiere.

\begin{tabular}{|ll|}
\hline 5101 & Antropología cultural \\
5101.01 & Adorno \\
5101.02 & Vestimenta \\
5101.03 & Danzas, fiestas (véase 6203.02) \\
5101.04 & Etnomusicología \\
5101.05 & Etnolingüistica \\
5101.06 & Museologia \\
5101.07 & Mitos \\
5101.08 & Magia \\
5101.09 & Poemas, relatos \\
5101.10 & Religión (véase 5403.04, 5506.20, \\
& $5601,5906.05,6301.10,7102.05$ \\
5101.11 & Hechiceria \\
5101.12 & Simbolismo (véase 6308.03) \\
5101.13 & Medicina tradiciona1 (véase 3209.04) \\
5101.14 & Tradición \\
5101.99 & Otros (especificar) \\
\hline
\end{tabular}

Ilustración 4: Subdisciplinas de Antropología cultural en las propuestas de 1973 y 1988 
La relación de subdisciplinas que los Códigos adjudican a cada una de las disciplinas antropológicas es arbitraria. Podemos preguntar qué argumento está detrás de la decisión de relacionar la Agricultura con la Etnología y no con la Antropología social, o por qué la Museología se vincula con la Antropología cultural y no con la Etnología. Como adelantábamos atrás, ¿es de hecho posible diferenciar las tres disciplinas de una manera clara? ¿No sería más adecuado distinguir las disciplinas antropológicas de una forma distinta?

Además, faltan por completo los ámbitos antropológicos que en los años 1970 y 1980 surgieron en una disciplina que intentaba abrirse a las nuevas condiciones y enfrentarse con los retos de las sociedades en un mundo cada vez más globalizado. Falta, por ejemplo, una subdisciplina que recoja la Antropología industrial y por extensión incorporar el enfoque dirigido a la cultura económica de las sociedades industriales, impulsado, en nuestro entorno, por investigadores como Esteve Fabregat con su obra Antropología industrial. ${ }^{16}$ Otro caso es la Antropología urbana, que si bien está presente en la propuesta de 1972, desaparece posteriormente. Y se trata de un campo que, debido a los procesos de éxodo rural en todo el mundo y las transformaciones en las ciudades, ha adquirido gran importancia en la labor antropológica. ${ }^{17}$ Parece que en los quince años trascurridos entre la primera propuesta que se presentó en 1973 y la versión final de 1988, los antropólogos perdieron el interés y/o no tuvieron capacidad de influir en una adaptación de los códigos.

No hay que olvidar tampoco a la Antropología física, que se ha incluido en otro campo (2402), en concreto, a Ciencias de la Vida. Por lo que la mayoría de las temáticas que aglutina se alejan de la Antropología, como por la Antropogenética, Antropometría, Antropología Forense, Osteología, o Somatología de los Primates y Biología Racial. Otras, sin embargo, sí formarían parte de nuestra disciplina: Archivos Antropológicos, Etnología, Hábitos Alimentarios, Museología y Antropología Médica.

Es significativo que la UNESCO abandone el proyecto en 1992, razón por la cual los Códigos UNESCO no se han actualizado desde entonces. Esto afecta de lleno a una disciplina tan dinámica y cambiante como ha sido Antropología social en los últimos, casi, 30 años. Es por ello que nos parece importante retomar la definición que contienen los Códigos UNESCO. Y no solo por su influencia en el

16 C. Esteva Fabregat: Antropología Industrial, Barcelona: Anthropos, 1984.

17 R. G. Fox: Urban anthropology: Cities in their cultural settings. Englewood Cliffs, NJ: Prentice-Hall, 1977. U. HANNERZ: Exploring the city: Inquiries toward an urban anthropology, New York: Columbia Univ. Press, 1980, (en español: Exploración de la ciudad. Hacia una antropología urbana, Madrid: Fondo de Cultura Económica, 1993). 
mundo académico español y la legislación española, sino también porque puede ser una herramienta para fomentar la reflexión. Es más, podría motivar a la UNESCO a retomar el proyecto. Por ello presentamos, a reglón seguido, los comentarios que hemos recogido entre colegas de la antropología como primera aproximación de la valoración de los Códigos UNESCO en nuestra disciplina.

\section{LOS CÓDIGOS UNESCO DESDE LA PERCEPCIÓN DE LOS/AS ANTROPÓLOGOS/AS ENCUESTADOS/AS}

Primero de todo, como ya hicimos en el breve texto introductorio que encabeza el conjunto de artículos vinculados al tema central de este número de la Revista Murciana de Antropología (Presente y futuro de la Antropología Social y Cultural española: A partir de las reflexiones sobre niveles de asociación científico-académica entre áreas de conocimiento), queremos expresar nuestro más sincero agradecimiento a los/as colegas, que desde distintas partes de la geografía española han participado en el pequeño cuestionario que les enviamos a propósito de sondear el asunto que nos ocupa. En concreto, por lo que aquí se trata, les preguntamos por su valoración sobre los descriptores de la disciplina en el Códigos UNESCO ${ }^{18}$ y, en caso de considerarlo necesario, que indicara las modificaciones a realizar.

Como exponemos a continuación, tres son los aspectos sobre los que fundamentalmente pivotan las respuestas del cuestionario, a saber:

1. El desfase de los Códigos UNESCO.

2. El desajuste de los Códigos UNESCO en relación a los contenidos que pretenden clasificar.

3. La necesidad de rehacer los Códigos UNESCO.

Los revisamos en este orden a partir de los comentarios de los protagonistas.

\subsection{El desfase de los Códigos UNESCO}

Respecto al desfase de los Códigos UNESCO es unánime el posicionamiento de los encuestados. De manera clara y concisa lo advierte Andrés Barrera González: «Esta clasificación de la UNESCO es cuando menos, por decirlo con palabras amables, anticuada e inconsistente»; Antonio Miguel Nogués Pedregal: «Muy antiguos, clásicos y tradicionales. Absolutamente desfasados»; y Enrique Perdiguero: «Pues, la verdad, creo que es una clasificación bastante superada pues está muy ligada a campos clásicos de la Antropología».

18 Se les remitió el documento Presente y futuro de la Antropología Social y Cultural española. 
Algunos llegan a calificar los códigos de decimonónicos, como hace Albert Moncusí Ferré: «Los descriptores remiten al modelo clásico de Antropología, sobre todo en lo referente a cuestiones de estructura social y política. (...) En definitiva, la clasificación adolece de una visión colonial decimonónica»; Juan Antonio Flores Martos: «Los descriptores en el Códigos UNESCO sobre nuestra disciplina, la antropología, se encuentran completamente desfasados, desmembrados y responden a una realidad de la antropología de finales del siglo XIX. La Antropología Social (y Cultural), o Antropología a secas, debería desembarazarse de parcializaciones y compartimentos estancos decimonónicos y poco operativos -más allá de la utilidad que tuvieron para el conocimiento enciclopédico y museográfico-museístico-, o como correspondientes a los códigos de las clasificaciones CDU en bibliotecas. Tuvieron su sentido y lógica, e inclusive operatividad, en el desarrollo de la disciplina en el pasado, pero carecen de sentido y utilidad en el presente», o Ángel Díaz De Rada: «Recogen una imagen de la antropología como disciplina de una alteridad exotizada, y de una especie de museografía decimonónica que ni siquiera representaría a la disciplina en elaboración en los tiempos de la "Etnografía" de Marcel Mauss, o de los Human Relation Area Files de Murdock - corrientes de diseño objetual de la disciplina ya en sí mismas con poca validez en nuestros tiempos, aunque mucho más elaboradas que esa lista arbitraria dispuesta en la codificación UNESCO». Es destacable lo mencionado por Flores Martos a propósito del sentido y valor que antaño pudieron tener los Códigos para museos y bibliotecas, o lo que señala Díaz de Rada cuando sugiere menor validez de los Códigos UNESCO que las «corrientes de diseño objetual de la disciplina» (ibid.) que derivan de los trabajos de Marcel Mauss y George Peter Murdock. En ambos casos se refuerza la idea de la actual obsolescencia de los Códigos.

David Florido del Corral conecta los Códigos UNESCO con los intereses de la Antropología en los años 40 y 60 del siglo pasado, fundamentalmente con las temáticas encasilladas entorno a las sociedades primitivas y los relacionados con la alteridad: «Considero que los descriptores responden a un modelo teórico y práctico de la antropología que no tiene sentido en la actualidad. Está claramente orientado al tipo de trabajo que se realizaba antes de la crisis de objeto y de método de la antropología, entre los años cuarenta y sesenta del siglo XX, pues refleja una clasificación que entiende la antropología como disciplina que se dirige al trabajo de las denominadas entonces "sociedades primitivas" y, metodológicamente, a la recolección de datos de determinados rubros desconectados, con especial incidencia en las dimensiones de la vida social que representan la "otredad" respecto a las sociedades occidentales modernas (mitos, magia, poemas y relatos, simbolismo, armas, trueque, caza, armas, forraje, jefatura y realeza, etc.)». 
Carmen Gregorio Gil se suma al planteamiento que exponemos en el documento de trabajo que les enviamos: «Coincido con lo que se propone en el documento en gran parte, ya que considero importante modificar los descriptores de la disciplina, en particular el código 5103 de la Unesco para adecuarlos a los profundos cambios experimentados en la disciplina y a las problemáticas y áreas de investigación de la antropología social contemporánea»; como también hace Manuel Moreno «Coincido plenamente con lo que se apunta en el documento Presente y futuro... en el sentido de que la descripción de los campos de trabajo está limitada y resulta un tanto obsoleta en relación a los cambios disciplinares producidos, fruto, a la vez, de los cambios en la sociedad».

\subsection{El desajuste de los Códigos UNESCO en relación a los contenidos que pretenden clasificar}

Aunque ya se adelantaba algún comentario sobre esta cuestión cuando se aborda en el punto anterior el desfase de los Códigos UNESCO, recogemos ahora respuestas más directamente relacionadas con el desajuste de los contenidos en los Códigos. Es recurrente la referencia a que quedan campos sin cubrir frente a otros que están en desuso, lo que genera una clasificación poco útil e imprecisa. Como señala Andrés Barrera González: «Combina en la elaboración de sus clasificaciones, de manera un tanto arbitraria y parcial, términos o criterios dispares referidos a: nombres de disciplinas, subdisciplinas, áreas de conocimiento, campos de estudio, con temas de investigación (ie. "jefatura y realeza", "trueque"). De lo que resulta un panorama confuso e inconsistente. El fundamento y rigor científico-académico de estas categorizaciones o clasificaciones es muy discutible, cuando no nulo». Añade, después Barrera otro comentario que nos parece clave, de hecho ha motivado el trabajo que tienen en sus manos. Lo expresa así: «En realidad, tales confusiones e inconsistencias se observan, en mayor o menor grado, en la mayor parte de las clasificaciones para fines académico-administrativos 19 que se manejan en España, desde las de la ANEP, a las de la ANECA... hasta las de la ACAP que menciona en su trabajo para la Universidad de Murcia».

Albert Moncusí Ferré también apunta en esta dirección, haciendo hincapié en la falta de encaje de categorías que son antropológicas por antonomasia: «Así, parecen de difícil encaje las categorías y diferenciaciones de género, generación, etnia/nación y clase, los movimientos migratorios y también las estructuras asociativas y los movimientos sociales y, en general, el análisis de todo lo relativo a

19 En las respuestas del cuestionario que nos ha remitido, Barreda González subraya «para fines académicos-administrativos». 
Estados modernos. Tampoco existen referencias a las nuevas tecnologías ni a la construcción del cuerpo, aunque esto último podría encajar en «Antropología médica». En la última parte de su alegato nos parece relevante cómo, ante la dificultad de encasillar temáticas, en concreto, «la construcción del cuerpo», sugiera la posibilidad de incorporarlo en una subdisciplina con la que puede tener relación («Antropología médica»). Entendemos que esta es otra señal de las limitaciones de los actuales Códigos UNESCO.

Enrique Perdiguero llama la atención sobre dos cuestiones muy relevantes y que nosotros también reflejamos en el primer epígrafe de este artículo. Nos referimos, de un lado, a los desajustes entre las subdisciplinas de Antropología social versus Antropología física; y de otro, al desligue de la Antropología social con la Etnología y la Etnografía. Perdiguero anota respecto a la primera cuestión que: «(...) quedando, especialmente infradesarrollado el referido a Antropología Social. Compárese, por ejemplo, el hipertrofiado desglose de los campos de Antropología Física con el que hay de Antropología Social. Es paradójico, hoy día, que la Antropología Médica este en el 1er campo y no en el segundo»; y con relación a lo segunda: «Tampoco está claro por qué hay que separar, desde un planteamiento actual, la Antropología social de los estudios etnográficos y etnológicos. Es obvio que esta clasificación recoge una realidad de la investigación antropológica verdaderamente periclitada y que recoge algunas tradiciones nacionales y otras no».

Carlos Diz Reboredo al abordar lo concerniente a los Códigos de la Antropología social también hace mención a la Antropología física, pero curiosamente, para «dejarla de lado»: «Parece evidente, dejando a un lado lo referente a la antropología física...», lo que también sugiere la existencia de la problemática entre la Antropología social y física que anteriormente reclamaba Perdiguero. Después, Diz Reboredo señala: «que muchos de estos códigos han sido pensados para una Antropología social muy determinada (en el tiempo y en el espacio). Muchos de estos códigos nos remiten a cuestiones que han sido enfocadas tradicionalmente en términos de "folklore", perfectamente válidos pero cada vez más distanciados de los ámbitos de la antropología contemporánea». En esta parte final de su reflexión abre la puerta de otro asunto que nos parece subrayable, el enfoque de los códigos de la Antropología social en términos de «folklore», lo que genera una brecha con las temáticas a las que actualmente se ocupa la Antropología.

En definitiva, podríamos resumir lo concerniente a los desajustes de los contenidos que los Códigos UNESCO pretenden clasificar, con los expuesto por Ángel Díaz De Rada «Esos códigos no ofrecen de ninguna manera una descripción de los objetos pertinentes para la antropología social y cultural contemporá- 
nea (y ni siquiera de los últimos cuarenta años)», y Manuel Moreno Preciado: «Los campos acotados se corresponden con temáticas que, o bien han perdido interés (soberanía, realeza, esclavitud...) o bien, han adquirido nuevas dimensiones (familia, parentesco, nomadismo...)»».

\subsection{La necesidad de rehacer los Códigos UNESCO}

En varias partes de este artículo insistimos en que los Códigos UNESCO son una herramienta fundamental en el ámbito académico español, a pesar, tal como estamos poniendo de manifiesto, de sus múltiples deficiencias. Sin duda esto debe invitar a su mejora, si bien, otra opción también factible, es dejar de usarlos. Aunque la mayor parte de las repuestas van en la primera dirección (los Códigos UNESCO han de rehacerse), hay también voces que no descartan apostar por su abandono. Tal como sugiere Ángel Díaz De Rada: «En mi opinión, la UNESCO debería revisar íntegramente esta codificación; aunque una solución más sencilla sería tal vez que nuestras instituciones dejasen de usar este absurdo instrumento como dispositivo de clasificación». Seguramente esta última reflexión derive de las continuas dificultades de su aplicabilidad en el quehacer académico cotidiano: «Cuando he tenido que usar esos códigos (por ejemplo, en el indexado de las tesis doctorales que he dirigido) han sido inútiles. De manera que, o bien he usado el descriptor más general (“Antropología Social” o "Etnografía”); o bien he recurrido al cajón de sastre "Otros"» (ibid.).

Como decíamos, la mayor parte de los encuestados apuntan por realizar cambios profundos en la clasificación UNESCO. Por ejemplo, Enrique Perdiguero lo expone así: «Sinceramente, creo que habría que plantear una reorganización genérica de toda la clasificación». Lo que coincide con la respuesta de David Florido que aboga por simplificar la clasificación englobando temáticas: «Entiendo que la mayoría de estos rubros son sustituibles, y englobables en otros de más alcance, que expresen las distintas dimensiones de la vida social, válidos para cualquier tipo de sociedad: sistemas simbólicos, sistemas económicos, sistemas de relaciones de poder, sistemas de organización social. Sólo así se expresa la posibilidad de comparabilidad entre modos de vida de sociedades, próximas y distantes».

Carlos Diz Reboredo, también apostando por la mejora de los Códigos actuales, sugiere, a diferencia de Florido del Corral, proponer nuevos códigos más ajustados a la realidad de la disciplina antropológica. Eso sí, subraya la imposibilidad de dar un código a cada subdisciplina. Lo plantea así: «En este sentido, no creo que sobren ni estén de más, pues afortunadamente muchas son hoy las antropologías que se piensan, se viven y se hacen, No obstante, de cara a actualizar los campos y los parámetros -en términos de Códigos UNESCO-, creo que una de las 
batallas habría de darse, precisamente, en la redefinición, o mejor, en el añadido de un conjunto de códigos más conectados con lo que actualmente se está haciendo en la disciplina. Obviamente, será imposible tener un Códigos para cada una de las subdisciplinas o para cada uno de los campos actuales, pero sí al menos proponer nuevos códigos para algunos de ellos. Cuestiones tan plurales como las relacionadas con los estudios del cuerpo y las sexualidades, el género, la memoria, la antropología política, las migraciones, los movimientos sociales, la ciencia y la tecnología, por citar solo algunos ejemplos, deberían poder verse reflejados en un abanico de códigos más amplio y diverso».

Uno de los encuestados, Juan Antonio Flores Martos, abre sus comentarios defendiendo la separación de la Antropología física o biológica para poder crear una única categoría, Antropología. Lo expresa de esta manera: «Desde mi propia experiencia como antropólogo en los últimos 20 años, propondría una sola categoría, diferenciada a su vez de la Antropología física o biológica, que podría denominarse «ANTROPOLOGÍA». ${ }^{20}$ Después sugiere que, a partir de esta gran categoría, se generen unos códigos específicos: «y para la que se podrían rescatar aquellos subcódigos, que tienen que ver directamente con las investigaciones actuales -además habría que incorporar otros subcódigos o códigos más específi$\cos$, que no figuran en la clasificación UNESCO-». Posteriormente presenta una relación de los mismos haciendo, en mayúscula, algunas aclaraciones: «Entre los rescatables estarían: 240206 Etnología, 240207 Antropología Médica, 240208 Hábitos Alimentarios, 5101 Antropología Cultural, 510103 Danzas, Fiestas (Ver 6203.06) (NO ENTIENDO LA AVERSIÓN A UTILIZAR LA CATEGORÍA «RITUALES»), 510104 Etnomusicología (ver 6203.06), 510105 Etnolingüística,_510106 Museología,_510107 Mitos,_510108 Magia,_510109 Poemas, Relatos (PODRÍA LLAMARSE «LITERATURAS INDÍGENAS»), 510110 Religión (ver 5403.04, 5506.21, 5601, 5906.05, 6301.10, $7102.05 \mathrm{y}$ 7204.04), 510111 Hechicería,_510112 Simbolismo (ver 6308.03),510113 Medicina Tradicional (ver 3209.04),_510114 Tradición, 5102 Etnografía y Etnología, 5103 Antropología Social, 510302 Filiación, Familia y Parentesco (QUE PODRÍA LLAMARSE AHORA «FAMILIA Y PARENTALIDADES»), ${ }^{21}$ 5199 Otras Especialidades Antropológicas (Especificar)». Finalmente, Flores Martos explica el sentido de su clasificación: «Lo que propongo es que este «cajón de sastre» de la nomenclatura y Códigos UNESCO -el 5199 «Otras Especialidades Antropológicas (Especificar)-, es prioritario y necesario el expandirlo con todas esas subdisciplinas y perspectivas de análisis que ha generado la

20 Flores Martos escribe Antropología en mayúsculas y negrita.

21 Todas las mayúsculas que aparecen en este verbatim son de Flores Martos. 
Antropología en los últimos 40-50 años: Antropología Aplicada (que inexplicablemente no figura en el Códigos UNESCO), Antropología del cuerpo, Antropología de las violencias, Antropología Económica, Antropología de las migraciones, Salud e interculturalidad, etc.».

Además, como sugiere Carmen Gregorio Gil (y nosotros subscribimos), la necesidad de rehacer los Códigos UNESCO no solo facilita clarificar los contenidos de nuestra propia disciplina, también es necesario para «establecer las áreas afines (que) nos ayudaría mucho en los diferentes procesos de evaluación, así como en la defensa de la profesión de antropólogo/a y en la configuración de los planes de estudios (materias básicas y optatividad)».

Como se desprende del documento Presente y futuro de la Antropología Social y Cultural española: A partir de las reflexiones sobre niveles de asociación científico-académica entre áreas de conocimiento, antes de conocer los distintos puntos de vista de los encuestados (que hemos presentado en este epígrafe), nos propusimos realizar una propuesta de clasificación de los Códigos UNESCO que mejorara la presente. Las reflexiones anteriores, aun con planteamientos diversos de los que nos hacemos eco, refuerzan nuestra inquietud por tal empresa. Y lo exponemos en las siguientes líneas.

\section{PRIMERAS PROPUESTAS PARA LA RECOMPOSICIÓN DE LOS CÓDIGOS UNESCO}

Como se ha visto en la segunda parte, hay una valoración unánime entre los participantes de la encuesta que califica los Códigos UNESCO como desfasados. Además expresan de manera clara y contundente la necesidad de elaborar una reforma de la nomenclatura.

Un proyecto con este objetivo no puede empezar de cero, tiene que partir de la consideración de que la Antropología social es una disciplina holística y abierta, tal como la describe Carmelo Lisón Tolosana: «Establecer fronteras rígidas es la antítesis de nuestra disciplina. Las manifestaciones de lo humano, siempre cambiantes, no lo permiten». ${ }^{22}$ Este carácter abierto, y quizás algo disperso, se refleja también en la reflexión realizada por Celeste Jiménez y Francisco Checa sobre los primeros treinta años de la revista Gazeta de Antropología, cuando, en relación a las temáticas abordadas por la Revista, señalaron que: «esa falta de puntos comunes, si ciertamente confiere un carácter heteróclito y quizá excesivamente disperso a la revista, desde otra perspectiva, sin embargo, ha posibilitado algunas de sus virtudes: la variedad de temáticas abordadas en los artículos, la apertura,

22 C. Lisón Tolosana (coord.): Antropología: Horizontes teóricos, Granada: Comares, 1998, p. 1. 
esa sensación de plena libertad y de carencia de censuras o cortapisas que tanto los autores como los directores han sentido, y de la que siguen gozando quienes publican en ella». 23

Tal como consta en esta cita, la Antropología se percibe como una disciplina en busca permanente de nuevos horizontes. Forma parte de su identidad y es una de sus características esenciales. No obstante, una nomenclatura exige fijar los ámbitos de la disciplina, aunque por supuesto tiene que ser un sistema siempre abierto a las pertinentes modificaciones.

Para realizar la labor de revisar la nomenclatura y proponer las modificaciones que creemos necesarias, nos valemos de una serie de reflexiones de antropólogos españoles sobre el carácter y el futuro de nuestra disciplina. Nos referimos especialmente a la compilación exhaustiva de publicaciones del ámbito antropológico que presentó Joan Prat en 1999. Contiene una sistemática de ordenación que (como señala Jesús Sanz Abad en una revisión de los grupos de investigación en el ámbito de la Antropología social) ${ }^{24}$ es una herramienta útil de partida para una tarea de este tipo. ${ }^{25}$ Además utilizamos varias obras introductorias o de reflexión sobre nuestra disciplina que están organizados de una manera parecida a la de Joan Prat y Ángel Martínez, ${ }^{26}$ y Carmelo Lisón Tolosana. ${ }^{27}$

Las categorías que utiliza Joan Prat en Investigadores e investigados son claras y evidentes. Su forma de ordenar la labor antropológica se basa, en la mayoría de los casos, en la distinción de «Antropologías de...». Seguramente Prat ha reproducido un formato utilizado, en tácita concordancia, por los antropólogos españoles durante las últimas décadas. Si esta es la formula a aplicar, nos cuestionamos lo siguiente:

¿Cómo podemos incorporar estos ámbitos antropológicos en la nomenclatura de la UNESCO?

¿Qué ámbitos de «Antropología de...» podemos diferenciar?

Respecto a lo primero habría que verificar cuáles son las disciplinas antropológicas, o dicho de otra manera, preguntarnos si sería adecuado considerar a la

23 C. Jiménez de Madariaga; F. Checa Olmos (2012): «Treinta años de Antropología en España. Memoria desde la Gazeta», Gazeta de Antropología, 2012, 28 (3), p. 9.

24 J. SANZ ABAD: «Panorámica general de los grupos de investigación en Antropología Social en el Estado español», Revista de Antropología Social, 20, 2011, pp. 9-29.

25 J. PRAT (coord.): Investigadores e investigados. Literatura antropológica en España desde 1954, Tarragona: Institut Tarragonès d'Antropologia (Arxiu d'Etnografia de Catalunya), 1999.

26 J. Prat; Á. Martínez (eds.): Ensayo de Antropología cultural. Homenaje a Claudio EstevaFabregat, Barcelona: Ariel, 1996.

27 C. Lisón Tolosana (coord.): Introducción a la Antropología social y cultural. Teoría, método y práctica, Madrid: Akal, 2007. 
Antropología cultural, a la Etnología y Etnografía, y a la Antropología social como las tres disciplinas que componen el campo Antropología. Además, si realmente se diferencian por contenidos y tradiciones diferentes, por qué no se ha establecido el Folklore como una disciplina propia, dado que desde el siglo XIX así es considerada. Por cierto, Folklore aparece en los Códigos UNESCO como subdisciplina de la Sociología cultural.

Como hemos señalado, a nosotros la diferenciación entre las tres disciplinas antropológicas de los códigos nos parece una distinción artificial que no refleja la realidad de la labor antropológica. Por esta y otras incongruencias, nuestra intención es presentar una propuesta de modificación que distinga las disciplinas antropológicas que se basan en sus diferentes objetos; las ramas de la labor antropológica que conocemos como «Antropología de...». Para ello sugerimos introducir estas ramas como disciplinas antropológicas en los Códigos UNESCO.

En relación a la otra cuestión (¿qué ámbitos de «Antropología de...» podemos diferenciar?) nos servimos, en una primera reflexión, de los ámbitos temáticos establecidos por Joan Prat (1999), un total de sesenta y seis. Por ejemplo: Antropología política, Antropología económica, Antropología urbana, Antropología simbólica...; donde también incluye: Artesanía, Cultural material, etc. Es por eso que nos planteamos si un número tan elevado es manejable a la hora de operar con ellos. Lo que nos encaminó a realizar un ejercicio de agrupación de disciplinas antropológicas que incluyan varias de las «Antropologías de...».

Con nuestra propuesta pretendemos iniciar una primera reagrupación de diferentes ámbitos antropológicos que conforman un total de diez disciplinas. Para ello hemos elegido entre los sesenta y seis ámbitos (de Prat), diez, y los hemos definido como disciplinas antropológicas (con cuatro dígitos), mientras que los restantes (más de cincuenta) aparecen como subdisciplinas (con seis dígitos). Las disciplinas son en su gran mayoría las que aparecen en obras introductorias como ámbitos más relevantes dentro de la Antropología. Esta labor nos ha llevado a configurar el siguiente listado, donde además añadimos, en notas al pie, cómo se denominan estos ámbitos en otras obras de referencia:

5101 ANTROPOLOGÍA COGNITIVA Y SIMBÓLICA:

Antropología cognitiva. ${ }^{28}$

Antropología de la comunicación.

Antropología lingüística.

28 Prat y Martínez (1996): Dominios cognitivos. 
Antropología de la religión. ${ }^{29}$

Antropología simbólica. ${ }^{30}$

Antropología visual.

Ideología. ${ }^{31}$

Lengua, Lenguaje.

\section{ANTROPOLOGÍA DEL CUERPO Y DE LA MEDICINA:}

Antropología de la alimentación. ${ }^{32}$

Antropología del cuerpo.

Antropología del deporte. ${ }^{33}$

Antropología de la medicina y la salud. ${ }^{34}$

Antropología de la muerte.

Antropología psicológica.

Antropología de la sexualidad. ${ }^{35}$

5103 ANTROPOLOGÍA DE LA DIFERENCIACIÓN SOCIAL: ${ }^{36}$

Amistad, Asociacionismo, Sociabilidad. ${ }^{37}$

Antropología de las edades. ${ }^{38}$

Antropología del género. ${ }^{39}$

Indigenismo.

Antropología de la marginación y desviación social. ${ }^{40}$

Antropología del parentesco y de la familia. ${ }^{41}$

29 Lisón Tolosana (2007): Religión y Creencia. Prat y Martínez (1996): Religiones.

30 Lisón Tolosana (2007): Rito, mito, símbolo. Prat y Martínez (1996): Símbolos.

31 Prat y MARTínez (1996): Ideologías.

32 Prat y Martínez (1996) aplican la misma denominación.

33 Prat y Martínez (1996): Antropología del deporte y del ocio.

34 Lisón Tolosana (2007): Antropología médica. Prat y Martínez (1996): Antropología de la salud.

35 Prat y Martínez (1996) aplican la misma denominación.

36 Esta denominación de un ámbito no aparece en Prat (1999) sino que está inspirada en PrAT y MARTíNEZ (1996). Lisón TOLOSANA (2007): Transformaciones. socioculturales y cambios familiares.

37 Prat y Martínez (1996): Amigos y vecinos.

38 Prat y Martínez (1996) aplican la misma denominación.

39 Lisón Tolosana (2007): Género y Antropología. Prat y Martínez (1996) aplican la misma denominación.

40 Prat y Martínez (1996): Antropología de la marginación.

41 Prat y Martínez (1996): Parientes y afines así como Antropología y transformaciones de la familia. 


\section{ANTROPOLOGÍA ECONÓMICA:42}

Antropología industrial (de la empresa...). ${ }^{43}$

Antropología de la pesca y marítima. ${ }^{44}$

Antropología del trabajo.

Antropología del turismo. ${ }^{45}$

Artesanía.

Campesinado, sociedad rural, agricultura. ${ }^{46}$

Cultura pastoril.

Recursos comunales.

\section{ANTROPOLOGÍA DE LA EDUCACIÓN47}

\section{ANTROPOLOGÍA DEL ESPACIO Y DEL TERRITORIO:}

Antropología ecológica. ${ }^{48}$

Antropología del espacio y del territorio.

Antropología urbana. ${ }^{49}$

5107 ANTROPOLGÍA DE LA MIGRACIÓN Y LAS RELACIONES INTERCULTURALES:

Antropología de la migración. ${ }^{50}$

Etnicidad, grupos y minorías étnicas, relaciones interétnicas.

Interculturalidad.

\section{ANTROPOLOGÍA POLÍTICA:51}

Antropología jurídica y de la ley.

Antropología política.

42 Lisón Tolosana (2007) aplica la misma denominación. Prat y Martínez (1996): Economía, cultura y cambio social.

43 Prat y Martínez (1996): Obreros así como Antropología de la empresa y también Antropología de los negocios.

44 Prat y Martínez (1996): Pescadores.

45 Prat y Martínez (1996) aplican la misma denominación.

46 Prat y Martínez (1996): Campesinos.

47 Tanto Lisón Tolosana (2007) como Prat y MARTínez (1996) aplican la misma denominación.

48 Prat y Martínez (1996): Ecología y cultura.

49 Tanto Lisón Tolosana (2007) como Prat y MARTínez (1996) aplican la misma denominación.

50 Lisón Tolosana (2007): Migración, sociedad y cultura. Prat y Martínez(1996): Antropología de las migraciones.

51 Lisón Tolosana (2007) aplica la misma denominación. 
Estudios de comunidad.

Identidad (identidad cultural, identidades colectivas, identidades étnicas...).

Nacionalismo.

Organización social.

Cambio (económico, social, cultural). ${ }^{52}$

5109 METODOLOGÍA, EPISTEMOLOGÍA, TEORÍA ANTROPOLOÓGICA:

Antropología aplicada. ${ }^{53}$

Antropología del discurso.

Antropología hermenéutica. ${ }^{54}$

Antropología de la historia.

Bibliografías y fuentes documentales.

Estudios comparados. ${ }^{55}$

Historia de la Antropología.

Historias de vida.

Metodología, epistemología y teoría antropológica

\section{PATRIMONIO ETNOLÓGICO Y CULTURAL:56}

Antropología del arte.

Antropología de la música.

Cultura material.

Cultura popular.

Cultura tradicional.

Fiestas. ${ }^{57}$

Folklore.

Literatura, literatura oral, narrativa. ${ }^{58}$

Museología y museografía.

Patrimonio etnológico y cultural.

Tradición oral.

52 Lisón Tolosana (2007): Cambios.

53 Lisón TOLOSANA (2007) aplica la misma denominación.

54 LisÓn TOLOSANA (2007): Giro interpretativo y reflexividad.

55 LisÓn TOLOSANA (2007): La comparación entre las culturas.

56 Lisón Tolosana (2007): Patrimonio cultural; Prat y MARTínEZ (1996): Antropología y patrimonio.

57 Prat y Martínez (1996) aplican la misma denominación.

58 Lisón Tolosana (2007): Literatura y antropología. Prat y MarTínez (1996): Narrativas. 
A lo visto, nuestro primer intento de ordenar la labor antropológica en disciplinas y subdisciplinas pretende seguir la filosofía de los Códigos UNESCO (con el uso de los cuatro y los seis dígitos). Considerando la dificultad que conlleva crear un sistema de disciplinas antropológicas, somos conscientes de las ambigüedades que contiene el listado propuesto. De modo que si, por ejemplo, hacemos referencia a la Antropología de la muerte, observaremos que está ubicada en la disciplina Antropología del cuerpo y de la medicina, pero podría estarlo en Antropología cognitiva y simbólica al contener la Antropología de la religión y estar muy vinculada con el ámbito anterior. Otro ejemplo que demuestra la dificultad de crear un sistema coherente es la ubicación de la Antropología ecológica. Nosotros la hemos integrado en la disciplina Antropología del espacio y del territorio, pero en muchos casos los antropólogos la definen como una parte de la Antropología económica. Además, nos hemos limitado a ordenar los sesenta y seis ámbitos mencionados por Prat ya en 1999. En nuestra encuesta han salido otros nuevos sobre cuya incorporación hay que debatir, como la Antropología de la violencia o la Antropología que versa sobre las (nuevas) tecnologías (por cierto, ya referida por Jesús Sanz Abad en 2011). ${ }^{59}$ Estos ejemplos demuestran que no hay una clasificación que recoja todas las sensibilidades y enfoques de las materias a ordenar, a la vez que la necesidad de seguir manteniendo el debate. Con todo esperamos que nuestra propuesta sirva para animar a que continúe y se avive la reflexión disciplinar. En realidad, la configuración del «listado» es una cuestión menor en comparación con la idea principal de la propuesta, que consiste en la recogida de los ámbitos antropológicos reconocidos en nuestra disciplina y su aprovechamiento para una reforma de los Códigos UNESCO.

Es la tácitamente reconocida estructuración de la Antropología en los diferentes ámbitos (disciplinas antropológicas) que señalamos, y con ello la propiedad intelectual colectiva, la que aquí reivindicamos para fomentar un proyecto común. Es por eso que entendemos que una reorganización de los Códigos UNESCO es necesaria y tiene cierta urgencia, dado, e insistimos en ello, que sigue siendo una de las herramientas básicas en la vida académica española para la definición de plazas, la descripción de tesis doctorales, o la adscripción a los grupos de investigación.

59 J. SANZ ABAD: «Panorámica general de los grupos de investigación en Antropología Social...», 2011, p. 20 


\section{BIBLIOGRAFÍA}

Jiménez De Madariaga, Celeste \& Checa Olmos, Francisco (2012): «Treinta años de Antropología en España. Memoria desde la Gazeta», Gazeta de Antropología, 2012, 28 (3).

Esteva Fabregat, Claudio: Antropología Industrial, Barcelona: Anthropos, 1984.

Fox, Richard Gabriel: Urban anthropology: Cities in their cultural settings, Englewood Cliffs, NJ: Prentice-Hall, 1977.

HANNERZ, Ulf: Exploring the city: Inquiries toward an urban anthropology, New York: Columbia Univ. Press, 1980 (en español: Exploración de la ciudad. Hacia una antropología urbana, Madrid: Fondo de Cultura Económica, 1993).

Lisón Tolosana, Carmelo (coord.): Antropología: Horizontes teóricos, Granada: Comares, 1998.

Lisón TolosAna, Carmelo (coord.): Introducción a la antropología social y cultural. Teoría, método y práctica, Madrid: Akal, 2007.

Ministerio de Ciencia, Innovación y Universidades del Gobierno de España: $<$ http://www.idi.mineco.gob.es/portal/site/MICINN/menuitem.8ce192e94ba 842bea3bc811001432ea0/?vgnextoid=363ac9487fb02210 VgnVCM100000 1d04140aRCRD\&vgnextchannel=28fb282978ea0210 VgnVCM1000001034 e20aRCRD>.

Ruiz Martínez, Juana María; Baños Moreno, María José \& Martínez BéJar, Rodrigo: «Nomenclatura Unesco. Evolución, alcance y reutilización en clave ontológica para la descripción de perfiles científicos», El profesional de la información, 23 (4), 2014, pp. 383-392

PRAT, Joan (coord.): Investigadores e investigados. Literatura antropológica en España desde 1954, Tarragona: Institut Tarragonès d'Antropologia (Arxiu d'Etnografia de Catalunya), 1999.

PrAT, Joan \& Martínez, Ángel (eds.): Ensayo de antropología cultural. Homenaje a Claudio Esteva-Fabregat, Barcelona: Ariel, 1996.

SANZ ABAD, Jesús: «Panorámica general de los grupos de investigación en Antropología Social en el Estado español», Revista de Antropología Social, 20, 2011, pp. 9-29.

UNESCO: Problems Encountered in the Development of a Standard International Methodology of Science Statistics, Meeting of the Adversory Panel of Science Statistics, UNESCO/CS/0666.SS-80/5, Paris, 6 June 1966.

UNESCO: Provisional List of Scientific Disciplines, UNESCO/NS/ROU/257, Paris, March 1972. 
UNESCO: Proposed International Standard Nomenclature for Fields of Science and Technology, UNESCO NS/ROU/257 rev. 1, Paris, 17 December 1973.

UNESCO: Proyecto de Nomenclatura Internacional Normalizada relativa a la Ciencia y la Tecnología, UNESCO NS/ROU/257 rev. 1, París, 17 de diciembre 1988 . 
